\title{
Methods of integrating the human nervous system with electronic circuits
}

\author{
Tymoteusz Skok ${ }^{1, A-F}$, Paweł Tabakow ${ }^{2, D-F}$, Krzysztof Chmielak ${ }^{2, D-F}$ \\ ${ }^{1}$ Student Scientific Circle at the Department of Neurosurgery, Wroclaw Medical University, Poland \\ 2 Department of Neurosurgery, Wroclaw Medical University, Poland \\ A - research concept and design; $B$ - collection and/or assembly of data; $C$ - data analysis and interpretation; \\ $D$ - writing the article; $E$ - critical revision of the article; $F$ - final approval of the article
}

\section{Address for correspondence \\ Tymoteusz Skok \\ E-mail: tymoteusz.skok@gmail.com}

Funding sources

None declared

Conflict of interest

None declared

Received on June 4, 2018

Reviewed on 0ctober 1, 2018

Accepted on January 28, 2019

Published online on August 12, 2019

\begin{abstract}
In recent years, many attempts have been made to connect electrical circuits with the human nervous system. The objective of type of research was diverse - from the desire to understand the physiology of the nervous system, through attempting to substitute nervous tissue defects with synthetic systems, to creating an interface that allows computers to be controlled directly with one's thought. Regardless of the original purpose, the creation of any form of such a combination would entail a series of subsequent discoveries, allowing for a real revolution in both theoretical and clinical neuroscience. Computers based on neurons, neurochips or mind prostheses are just some examples of technologies that could soon become part of everyday life. Despite numerous attempts, there is still no interface that meets all the expectations of the scholars. However, many scientific groups seem to be on the right track and their achievements raise extraordinary expectations. This paper evaluates historical theories and contemporary ideas about such interfaces to smoothly describe the major medical and scientific utility of the subject. Thus it presents the main issues surrounding the concept of integrating the human nervous system with electronic circuits.
\end{abstract}

Key words: brain-computer interface, biosensors, peripheral nerve interface

Cite as

Skok T, Tabakow P, Chmielak K. Methods of integrating the human nervous system with electronic circuits. Adv Clin Exp Med. 2019;28(8):1125-1135. doi:10.17219/acem/103414

DOI

10.17219/acem/103414

\section{Copyright}

Copyright by Author(s)

This is an article distributed under the terms of the

Creative Commons Attribution Non-Commercial License

(http://creativecommons.org/licenses/by-nc-nd/4.0/) 


\section{Introduction}

The first historical attempts to interpret the relationship between mind and body were undertaken by Alexandrian doctors around $250 \mathrm{BC}$. They concluded that "pneuma psychikon" ("mental spirit") moves along the nerve fibers, which stimulate the muscles of the animals to contract. This theory was taken up by Galen, who included it in his works on the subject of the human body. For the next 1,500 years, European medicine did not try to oppose any of his theses. ${ }^{1}$ In 1664, Jan Swammerdam made a breakthrough discovery in the field of neurophysiology. He discovered that the muscle of a dissected frog retains the ability to contract post mortem and that this contraction can be induced by irritating the nerve connected to the muscle. This discovery led to the demise of current views on the induction of motor reactions. ${ }^{2}$ Thus, the "pneuma psychikon" theory was renounced by the scientific community. However, Swammerdam was not able to answer how the nerve irritation causes the muscles to contract. ${ }^{3}$

In 1713, Isaac Newton suggested that this phenomenon could be related to electrical activities occurring in the living organism. The final scientific evidence for the existence of electrical phenomena in tissues was described at the end of the $18^{\text {th }}$ century. In 1791, Luigi Galvani proved that the stimulation of the muscle in Swammerdam's experiment is associated with the conduction of an electrical impulse. ${ }^{4}$ A new field of science, electrophysiology, was established. A systematic description of how electrical impulses control processes occurring in living organisms was performed. In 1825, Leopoldo Nobili invented the first galvanometer, which allowed him to study in detail the electrical phenomena in tissues. Subsequent discoveries by Helmholtz, Bernstein and Overton made it possible to determine the features that characterize neuromuscular agitation. ${ }^{5}$ The work of Ramón y Cajal, Berger and Cole later laid the foundations for modern methods of perceiving the electrical activity of the human nervous system.

The real revolution, however, began with the development of the voltage-clamp method by Cole and Marmont. ${ }^{6}$ Thanks to this technique, Hodgkin and Huxley investigated the mechanisms of electrical activity in neurons, for which they also received the Nobel Prize in 1963. Their work enabled a whole range of discoveries related to the physiology of propagation of electrical arousal in neurons. Simultaneously with electrophysiology, a completely new trend developed in culture and science. In 1911, Jean de la Hire in his novel "Le Mystère des XV" presented the concept of connecting man with a machine. This idea was quickly picked up by other science-fiction writers. It has resulted in numerous stories, novels and films about the use of artificial implants in the human body. At the same time, Pierre Teilhard de Chardin, in his book "The Future of Mankind" (1949), used, for the first time, the term "transhumanizing". It meant raising, with the help of technology, the potential of human capabilities to the maximum level. Soon, the concepts of improving the human body, including the nervous system, were collectively referred to as transhumanism. With the emergence of this concept, a group of philosophers and ethicists started considering the possible consequences of the development of such a type of technology. ${ }^{7}$

The first idea to use an electrical system to eliminate a human body defect precedes the book of de Chardin. Research conducted in 1940 by Jones, Stevens and Lurie made it possible to hypothesize the possibility of using electrostimulation in the case of damage of the cochlea. Thanks to this, Djourno and Eyriès decided to conduct a revolutionary operation. In 1957, they managed to connect the $8^{\text {th }}$ cranial nerve (vestibulo-cochlear) with an electrode, and thus to perform the first cochlear implant insertion surgery. It was not only the first electric auditory implant, but also the first successful electrical implant in general. ${ }^{8}$ Since that time, many concepts have been created in the field of electronics implementation in human tissue.

At the beginning the $21^{\text {st }}$ century, Grattarola laid the foundations of a new branch of science - neuroengeneering. In 2003, the first international conference on neuroengineering was held, and in 2004 the first neuroengineering journal - "The Journal of Neural Engineering" - was established. Neuroengineering is a science on the border of neurobiology, physics, mathematics, electronics, and bioengineering. Creating an interface between nerve tissue and an electrical circuit is just one of many issues that this field deals with. Other issues related to neuroengineering are techniques of the stimulation of nervous tissue regeneration, neuroimaging, the creation of mathematical models of neural network behaviors, and many more. ${ }^{9,10}$

The aim of this paper is to present the history and current knowledge and experience in the field of research on the application of methods to connect the human nervous system to artificial electric circuits.

\section{Material and methods}

During the development of this paper, the data described was acquired mainly through the use of the PubMed search engine. Other search engines, such as: ScienceDirect, Nature and Google Scholar, played a supportive role in situations when the authors were interested in finding a very particular article, and it was not included in the PubMed database.

To explore the subject, the authors set a number of issues to be explained in terms of every method described. They formed those issues afterwards in the following list of questions:

- Which ideas had already been proposed to integrate nervous tissue with electrical circuits?

- Was such an idea realized? If so, did it meet all the goals set by the authors of the technique? 


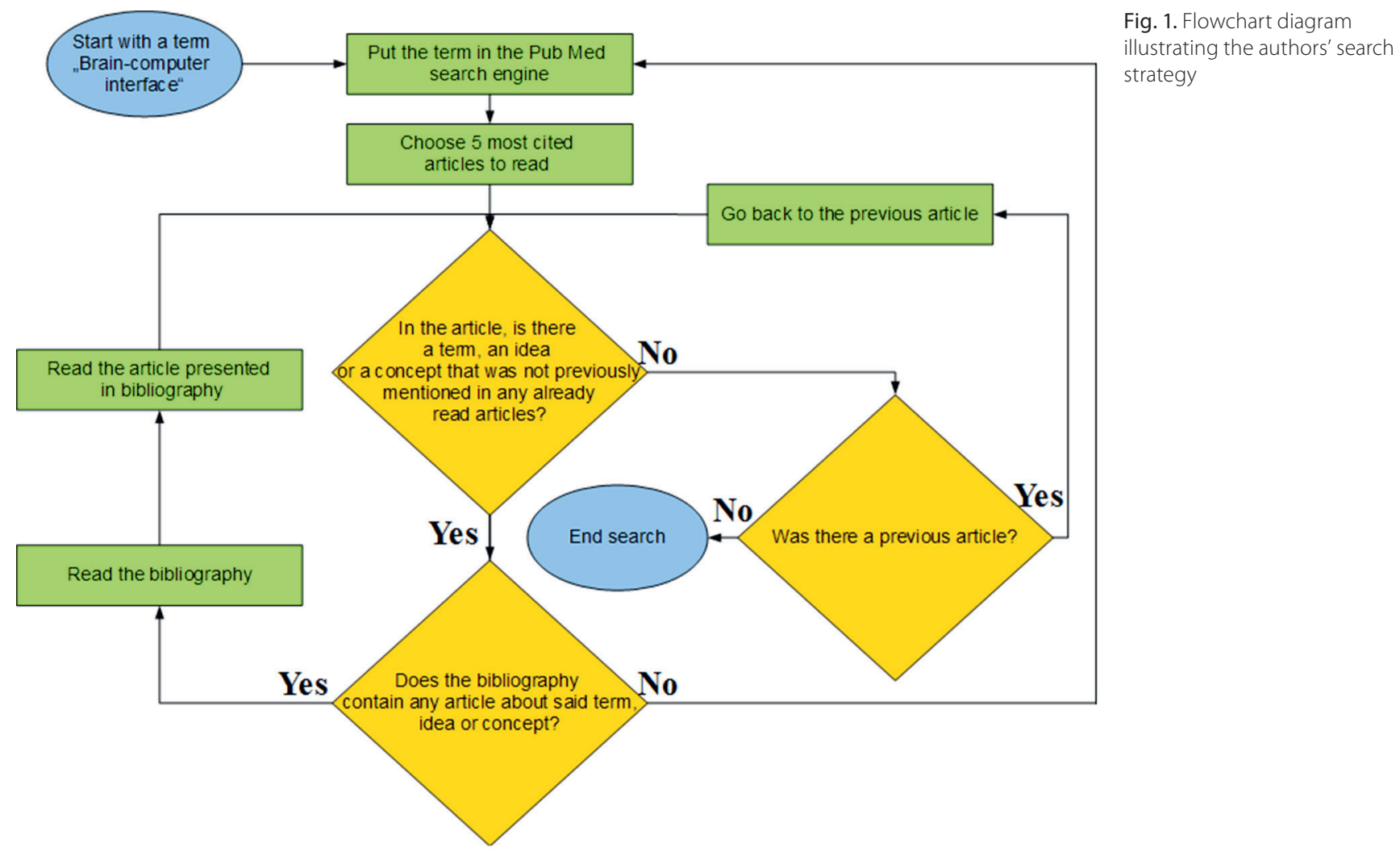

- If it didn't, what are the restrictions of this technique? Has the idea been further developed? What are the predictions for development of the technique?

The lack of answers in newly reviewed papers created a need for further research. The authors were looking for new articles, based on the bibliographies of the previously evaluated papers and many search terms aimed particularly at this task.

During the construction of the bibliography, the authors have tried to include papers meeting as many of the following criteria as possible: original paper; answers at least 2 of the problems mentioned above; greatest possible applicability of the described techniques; most detailed description of the topic; accurate, methodological results. The algorithm guiding the authors during material collection is presented in Fig. 1.

\section{Results}

\section{Paper search}

The PubMed, ScienceDirect, Nature, and Google Scholar databases were searched. Over 20,000 articles were considered for review during the research. After a selection based on the number of citations, 120 articles were reviewed and 60 have been included in bibliography. The material described was collected both from original papers and other review articles. This allows the reader to examine various insights on the subject of the work.

\section{Basic principles of electrophysiological data processing}

Most of the techniques described in this article are based on electrical readings measured with electrodes. Neurons, depolarizing, elicit an electromagnetic field around them. This field can stimulate electrons in electrodes to move, thus creating an electric current. The individual properties of an electrode, such as proximity to the signal and internal impedance, have an impact on the ability to record those arousals. Therefore, depending on the method, the quantity of impulses noticed by the electrode may vary in number. Its reading capacity can range between a single neuron, a small group of cells and even thousands of neurons. Such data can be further processed peripherally (for example by a differential amplifier), and then they can be directly coded in the form of digital records. One of many popular devices for such translation is, i.e., the open source Open BCI platform. Information is allocated in the primary storage of the computer, where it can be further used as a mathematic variable. Computer software can process this data in any way the user wants. For example, for EEG readings, there are many software packages that enable easy data processing, like EEGLAB (Swartz Center for Computational Neuroscience, University of California at San Diego, USA) or the Neurophysiological Biomarker Toolbox (VU University Amsterdam, the Netherlands). It is also possible to reverse the way of signal conduction. The effect would be generation of an electromagnetic field around the electrodes, thus facilitating the depolarization 
in nearby neurons. This is how afferent stimulation of the nervous system could be evoked. Therefore, appropriate programs for electrode firing patterns can elicit an artificial feeling of sensation or stimulate body effectors (muscles) to perform complex movements.

\section{Technology review}

In general, the technology that connects the nervous system with electronic systems can be divided into 3 large categories:

- devices that record and control pulse transmission in the cerebral cortex and the central nervous system (BCI - brain-computer interface);

- devices that record and control the transmission of nerve impulses in the peripheral nerves (PNI - peripheral nerve interface);

- biosensors that record nerve cell activity in vitro.

Table 1. Possible medical use of electronic interfaces for nerve tissue

\begin{tabular}{|c|c|c|}
\hline $\begin{array}{l}\text { Interface } \\
\text { type }\end{array}$ & Condition & Mechanism of action \\
\hline $\begin{array}{l}\text { Non-invasive } \\
\mathrm{BCl}(\mathrm{EEG})\end{array}$ & $\begin{array}{l}\text { lock-in syndom } \\
\text { spinal cord injury }\end{array}$ & $\begin{array}{l}\text { general control of external } \\
\text { devices, like cursor on computer } \\
\text { screen }\end{array}$ \\
\hline \multirow{4}{*}{ Invasive $\mathrm{BCl}$} & \multirow{2}{*}{$\begin{array}{l}\text { lock-in syndom } \\
\text { spinal cord injury }\end{array}$} & $\begin{array}{l}\text { precise control of external } \\
\text { devices, like robotic arms }\end{array}$ \\
\hline & & $\begin{array}{l}\text { bypassing injury by direct } \\
\text { motoneuron or muscle } \\
\text { stimulation }\end{array}$ \\
\hline & $\begin{array}{l}\text { visual or auditory } \\
\text { impairment }\end{array}$ & $\begin{array}{l}\text { restitution of visual or auditory } \\
\text { function }\end{array}$ \\
\hline & direct brain injury & $\begin{array}{l}\text { replacement of the missing } \\
\text { brain tissue (so far only } \\
\text { hippocampal injuries) }\end{array}$ \\
\hline PNI & limb amputation & $\begin{array}{l}\text { interfaces for high quality } \\
\text { prosthesis control }\end{array}$ \\
\hline
\end{tabular}

$\mathrm{BCl}$ - brain-computer interface; EEG - electroencephalography; $\mathrm{PNI}$ - peripheral nerve interface.

\section{Brain-computer interface}

The term BCI was used for the first time by Jacques Vidal in 1973. In his work "Toward direct brain-computer communication", he described how, using an EEG apparatus, a machine could hypothetically read a brain's decisionmaking process. He later constructed a system in which the respondent, with the help of evoked potentials, could control the appropriate symbol on the screen of a monitor. The subject was also able to guide the cursor through a simple maze puzzle. ${ }^{11}$ The concept created by Vidal was developed and expanded in the following years. New trends appeared in the field of BCI technology. Contemporary brain-computer interfaces are not just machines based on EEG. The name BCI is actually defined as any device capable of collecting, analyzing and processing data directly from the brain. ${ }^{12}$ Recently, a huge number of different concepts, projects and plans for BCI have been created. Bringing them all together in one article seems unrealistic. Therefore, only the dominant ideas and trends in the field of BCI technology will be discussed. Brain-computer interfaces are usually divided according to their invasiveness. ${ }^{13}$ They can be non-invasive, partially-invasive and invasive.

\section{Non-invasive brain-computer interface}

Since 1970s the use of electroencephalography remains the most popular solution in the field of non-invasive BCI. This is mainly due to purely technical issues. Modern brainwave-reading devices can have the form of only several electrodes, which can be attached to the surface of the head. This makes them much handier and more practical than sophisticated research equipment. Connecting the EEG to the subject can take only a few minutes. Thus, the simplicity and convenience associated with the EEG tempts many researchers to use it in BCI. In recent years, a whole multitude of devices and instruments have been created. They allow, among others: writing on a computer screen, ${ }^{14}$ wheelchair control ${ }^{15}$ and web browsing. ${ }^{16}$ They can even be used purely for entertainment purposes, such as playing computer games. ${ }^{17}$

The EEG-BCI technology still leaves much to be desired. Its biggest problem is the relatively low sensitivity and specificity, resulting in an inability to unambiguously read the signals sent by the subject. The relatively large number of errors made by the interface makes it impossible to use it in everyday life. ${ }^{18}$ Nevertheless, work is ongoing on improving the methods of reading and interpretation, which may lead to the elimination of these drawbacks in the near future.

Other ideas in the field of non-invasive BCI are interfaces based on magnetic resonance imaging (MRI) and magnetoencephalography (MEG). The MRI-BCI technique has significant limitations. It has not yet been determined how the specific cognitive states of the brain could be read using an MRI machine. In addition, the tests may only be carried out in facilities with a resonance imager. This is a bigger problem, because the price of this type of equipment significantly limits its availability and universality. Despite this, attempts are still being made to implement fMRI-BCI technology in medicine. So-called "real-time MRI feedback" is sometimes used in post-stroke rehabilitation (learning how to reactivate subsequent levels of the pyramid system) as well as in teaching patients how to cope with pain and emotions. ${ }^{19}$

Magnetoencephalography (MEG) carries disadvantages similar to MRI. Large and cumbersome equipment generates the readings, the understanding of which still causes significant difficulties for modern science. At the same time, like the other interfaces of this group, the MEG apparatus is very imprecise and the subjects need a lot of time to learn how to use it. Most likely, MEG technology will not lead to the creation of a useful BCI in the near future. ${ }^{20}$ 


\section{Partially-invasive brain-computer interface}

Current semi-invasive methods of BCI interface implantation are basically limited only to electrocorticography (ECoG) methods. Although ECoG is a method very similar to the EEG, the electrodes are located not on the surface of the patient's skull, but directly in the epidural or subdural space. This has many advantages over the EEG. ${ }^{21}$ Electrocorticography represents a much higher resolution, higher amplitude of changes, and therefore greater sensitivity, higher bandwidth and smaller susceptibility to electrical interference due to muscle contraction and eye movement. However, semi-invasive interfaces have also significant limitations. Their basic disadvantage in comparison to EEG-BCI is the need to carry out operations with a risk of undesirable adverse events. Nevertheless, ECoG-BCI continues to be considered a promising technology. It does not come into direct contact with the cerebral cortex. This makes the microglial reaction and the formation of glial scars around electrodes delayed in time and less prominent (as in the case of fully invasive interfaces), allowing for a longer viability of the device (Fig. 2).

\section{Invasive brain-computer interface}

Invasive $\mathrm{BCI}$ allows the most detailed perception of electrical activity in the human central nervous system. Depending on the spatial resolution of the recorder, 2 types of invasive BCI can be distinguished: multi-unit activity (MUA) recording and single-unit activity (SUA) recording. The concept of SUA recording seems to be the most exciting. This is a technology in which individual electrodes would be able to contact and cooperate with single neurons. Unfortunately, currently, it is not possible to create a BCI consistent with such an idea. It seems, however, that

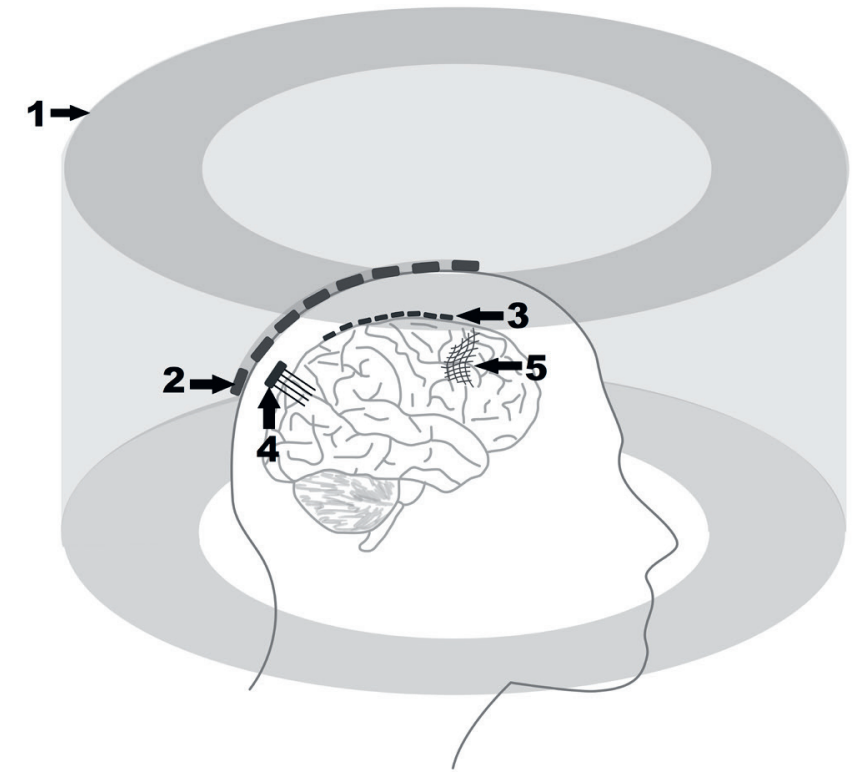

Fig. 2. Different approaches to brain-computer interface: 1. Magnetoencephalographic $\mathrm{BCl} /$ magnetic resonance $\mathrm{BCl}$; 2. Electroencephalographic $\mathrm{BCl}$; 3. Electrocorticography $\mathrm{BCl}$; 4. Multi-unit activity recording $\mathrm{BCl}$; 5 . Single-unit activity recording $\mathrm{BC}$ the possibility of creating a direct connection between a neuron and electronics is heralded by the emergence of so-called neural mesh technology. It involves injecting electrodes into the cerebral cortex, which, slowly developing, over time would be able to come into contact with each neuron individually. ${ }^{22}$

In MUA recordings, electrodes interact with whole groups of neurons in their vicinity. Devices of this type have already succeeded in supporting patients with tetraplegia, lock-in syndrome or other neurological deficits. In 1998, Philip Kennedy constructed the first machine that was directly connected with the human brain. Based on principles of neural regeneration and low impedance recording, he used a glass tube with neurotrophic factors and wires made out of gold to achieve a stable BCI interface (the so-called neurotrophic electrode). During his test with a patient suffering from severe amyotrophic lateral sclerosis, he managed to implant his system in the human motor cortex. Thus, he created an effective way of communication between the patient and his environment. ${ }^{23}$ A similar concept has been used in the Brain Gate project - a venture that was created to enable paralyzed people more effective communication with the surrounding world. ${ }^{24}$ Brain Gate decided to use a pattern of stable, numerous needle-like electrodes to establish a connection between the motor cortex and a computer (the Utah electrode array). The aim of the Brain Gate project was to give patients with lock-in syndrome the ability to communicate with the environment using the cursor on the computer screen or artificial, robotic arms. Other research groups soon followed the steps of Brain Gate and since 2002, there have been many projects whose aim was to allow neuroprotheses to be controlled by people with tetraparesis ${ }^{25,26}$ (Fig. 3).

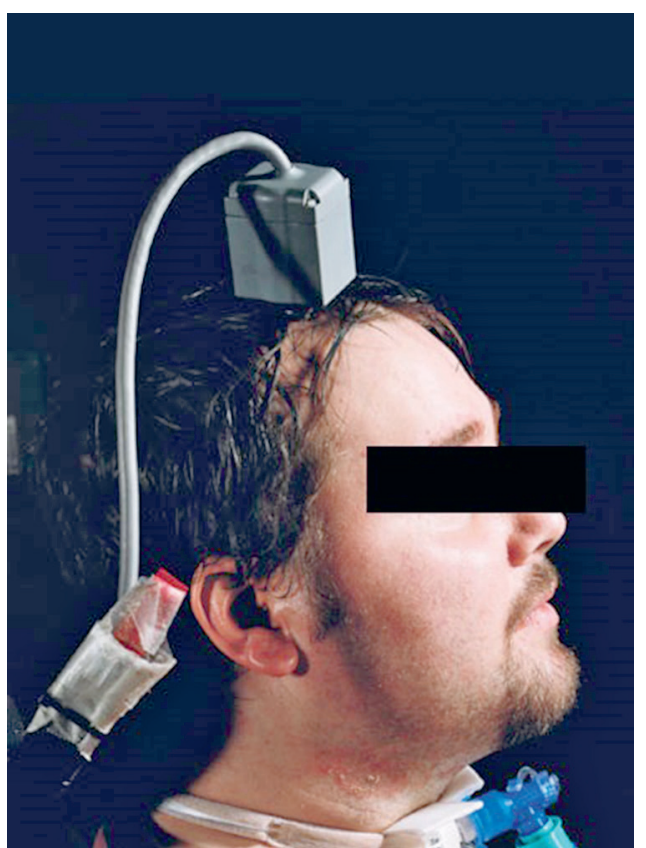

Fig. 3. Matthew Nagle, the first paralyzed person that ever used Brain Gate technology ${ }^{27}$ 
Although BCI-controlled artificial robotic limbs are a great tool to improve the quality of life of patients with spinal cord injury, an alternative therapeutic concept for these patients has recently been developed. It is based on a method of direct wireless transmission from the brain of signals for voluntary contraction of the paralyzed muscles by bypassing the area of spinal cord injury. Capogrosso et al. have managed to record signals both from motor cortex and spinal motoneurons in primates. ${ }^{28}$ By optimizing the data, they recreated a spinal cord motoneuron firing pattern, and ordinated it with signals from the motor cortex. The Wi-Fi microarray receptor in the head and epidural electrical stimulator implanted in the lumbar spinal cord locomotor generator of a monkey made it possible to alleviate gait deficits after spinal cord injury. ${ }^{28}$ Another approach was proposed by a research group from Columbus, USA. Their idea was not concentrated on translating brain signals to spinal cord motoneurons, but directly to the patient's muscles. This enabled a 24-year-old C5/C6 quadriplegic patient to restore the ability for voluntary movement of his wrist and hand. He was even able to perform complex tasks, like playing on a computer game controller or pouring dice from one cup to another. ${ }^{29}$ Invasive BCI are also capable of replacement of neurons in the lesioned central nervous system (CNS). An example of such an application is the treatment of visual deficits by application of prostheses activating the visual cortex. By implantation of the electrodes on the occipital lobe, William Harvey Dobelle caused electrical stimulation in the appropriate areas of the cortex, thus evoking visual sensations in the subject. This enabled blind people to experience a complete sense of vision. By combining this type of interface with a camera, Dobelle helped blind people to function more freely in society. ${ }^{30}$ Although there are more contemporary ideas of visual function supplementation, so far only 2 projects have received the CE mark for use in Europe - Argus II and Alpha IMS. Argus II is an artificial implant that, in response to light received by a camera, stimulates optic nerve and remaining retina cells. ${ }^{31}$ Subretinal Visual Implant Alpha IMS, on the other hand, detects light stimulus directly inside the eye. Subsequently, it activates the retina's bipolar cells, creating a sensation of seeing. ${ }^{32}$

Another example of the usage of invasive BCI for the replacement of structures of the CNS is the project developed by Theodor Berger. It involves the creation of a prosthesis that would correspond to the function of the lesioned hippocampus. It is supposed to help people with memory deficits due to hippocampal lesion. ${ }^{33}$

Invasive $\mathrm{BCI}$ also have disadvantages. As an invasive method, they may require complex surgeries with risk of complications. The main concern about invasive BCI is the fact that glial scarring develops over time, preventing the reception of signals. ${ }^{34}$ In conclusion, a comparison of non-invasive and invasive BCI shows that there will be an indication for both technologies. It is highly probable that non-invasive $\mathrm{BCI}$ will never be able to match invasive in its detail and functionality. The EEG-BCI is still likely to be used in the fields of neurorehabilitation. With the development of science and the emergence of ever-better systems, it seems that invasive BCI will overcome noninvasive BCI in most applications, certainly those associated with the restoration of motor functions. ${ }^{35}$

\section{Peripheral nerve interface}

Peripheral nerve interface (PNI) allows a registration of electrical stimuli in peripheral nerves. ${ }^{36}$ The technology boom associated with PNI began around the 1990s and is still developing. There are more and more new concepts on the methods of electrode implantation, the types of used materials and the ability to enhance the signal coming from the axons. The ideas around peripheral nerve interfaces are mainly focused on creating an interface for intuitive use of artificial limb prostheses. There are 3 basic approaches attributed to PNI: external nerve PNI, intranervous PNI and regenerative PNI. ${ }^{37}$

\section{External nerve peripheral nerve interface}

Nerve cuffs (Fig. 4A) is the most basic approach in the field of PNI. They are electrodes, attached to the surface of the nerve, so as to perceive its internal electrical activity. ${ }^{38}$ There are many models of these devices, including cylindrical, helical and annular types. They all have a low specificity in recognizing nerve stimuli. Moreover, poor implementation of such an interface, as a result of nerve compression, manifests itself with a strong reaction from the connective tissue of the epineurium. This leads to quick scar formation and destabilization of the interface. ${ }^{39}$

The epineurium surrounding individual nerve fascicles is a relatively compact tissue, while the perineurium connecting these fibers with each other is relatively loose and plastic. This has made it possible to create a flat-interface nerve electrode (FINE) interface, which, when implemented on the nerve, changes its architecture (Fig. 4B). The nerve takes the form of a flat strip with nerve bunches placed flat next to each other. In this way, individual electrodes are able to study the electrical activity in the corresponding parallel tufts. At the same time, with the right choice of parameters, FINE does not adversely affect the physiological and histological features of the nerve. ${ }^{40}$ Tests to use this technology in medical practice are still ongoing. ${ }^{41}$

\section{Intra-nervous peripheral nerve interface}

The classic intra-nervous types of PNI include such projects as TIME (transverse intra-fascicular multichannel electrode) (Fig. 4C), LIFE (longitudinal intra-fascicular electrode) (Fig. 4D) and SPINE (slowly penetrating interfascicular nerve electrode) (Fig. 4E). Longitudinal intrafascicular electrode and TIME are longitudinal electrodes, threaded with needles directly in the nerve structures. Longitudinal intra-fascicular electrodes are arranged in parallel along the nerve fascicle, according to the course 

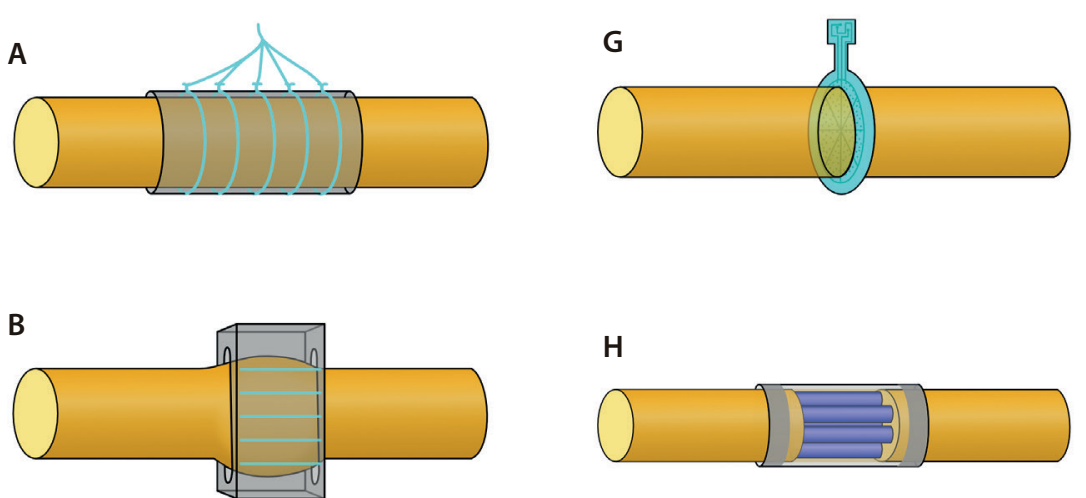

H
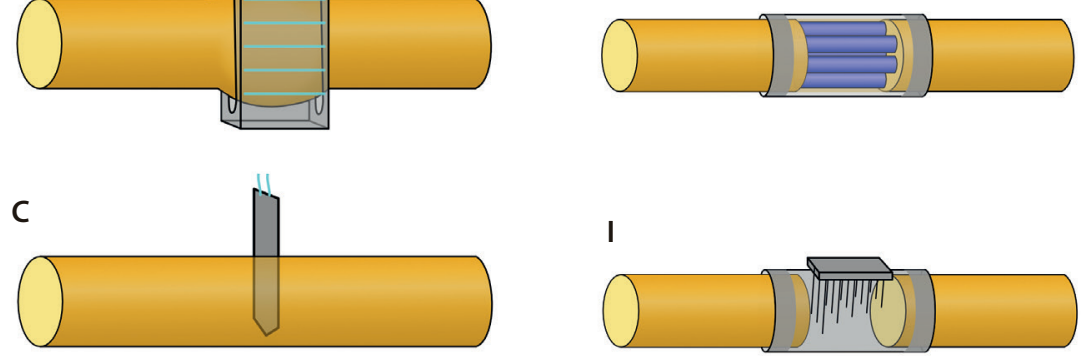

I

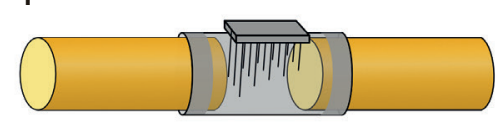

D

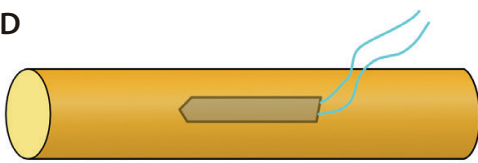

E
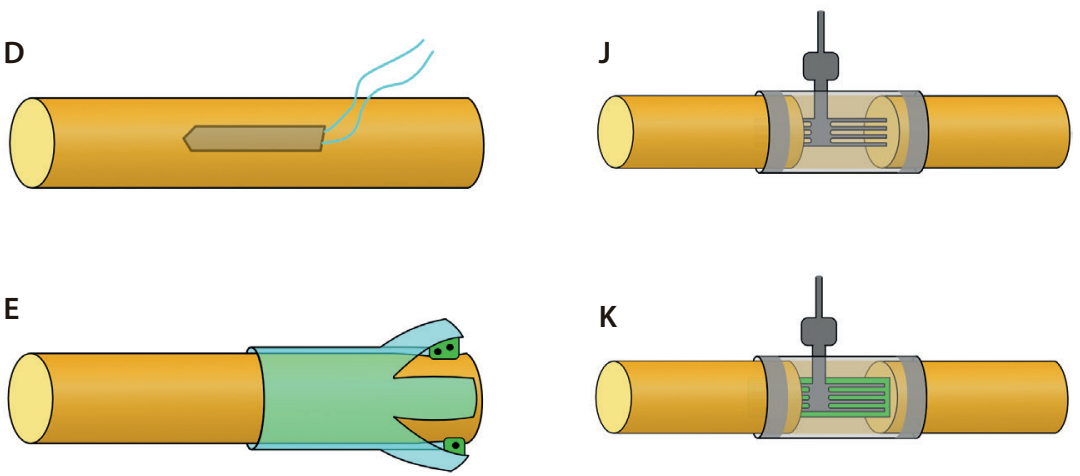

Fig. 4. Different approaches to peripheral nerve interfaces: A. Nerve cuffs; B. FINE (flat-interface nerve electrode); C. TIME (transverse intra-fascicular multichannel electrode); D. LIFE (longitudinal intrafascicular electrode); E. SPINE (slowly penetrating interfascicular nerve electrode); F. USEA (Utah slant electrode array); G. SNI (sieve-nerve interface); H. MNI (microchannel-nerve interface); I. REMI (regenerative multielectrode interface) J. RSE (regenerative scaffold electrode); K. TEENI (tissue-engineered electronic nerve interface)

The USEA (Utah slant electrode array) (Fig. 4F) interface is composed of 100 electrodes, $0.5-1.5 \mathrm{~mm}$ long. Each of them is mounted on a 10-electrode array of 10 rows. The USEA is placed on the nerve by puncturing the peri- and epineurium with electrodes. In this way, each electrode offers a channel for the perception of electrical activity in the appropriate nerve compartment. Despite the relative simplicity of the idea, the USEA seems to be very successful in clinical trials. In the tests conducted on patients after amputation, the subjects were able to smoothly move the virtual hand on the monitor screen. It even managed to evoke the sensation of touch with the help of electric stimuli. ${ }^{46}$ It is also worth mentioning that this model of electrode system is also used in invasive BCI techniques. It has been used, among others, in the aforementioned Brain Gate project.

Sieve-nerve interface (SNI) (Fig. 4G) and microchannel-nerve interface (MNI) (Fig. $4 \mathrm{H}$ ) are technologies on the border of the nerve. ${ }^{42}$ Transverse intra-fascicular multichannel electrodes are implanted perpendicular to the nerve. They pierce the bundles through, transversely across the entire width of the nerve fascicle. ${ }^{43}$ In SPINE, blunt electrodes press slowly on epineurium, squeezing into the nerve. Simultaneously, they rearrange nerve architecture to contact the largest possible number of nerve fibers. The changes in the mutual position of the fibers were to facilitate effective signal reception through the interface. ${ }^{44}$ Unfortunately, it is difficult to find a modern publication on this technique. Most likely, it suffered from the same condition that TIME and LIFE suffer to this day. As a result of irritation of nerve structures around the electrodes, a scar made of glial cells arises over time. This makes the interface unresponsive to arousal and therefore unusable. Despite this, attempts are still being made to implant these types of electrodes. The use of more advanced artificial materials and biomaterials raises the hope of eliminating the phenomenon of the response of the body to the electrode. ${ }^{45}$ of invasive and regenerative PNI. The SNI is a transverse intersection of the nerve, followed by implantation of the SNI, consisting of electrodes, in the place of the nerve cut. ${ }^{47}$ Regenerating, single axons pass through the mesh of the sieve, which allows the perception of the potentials generated by them. The MNI is not much different from this approach. According to Musick et al., ${ }^{48}$ regenerative microchannel electrodes are essentially long sieve implants that host millimeter lengths of the nerve. Both concepts are very invasive and often lead to intensive foreign body response reactions. A summary of the peripheral nerve interfaces used is shown in Fig. 4. A comparison of different types of PNI in reference to selectivity and interface invasiveness is shown in Fig. 5.

\section{Regenerative multielectrode interface}

Regenerative multielectrode interface (REMI) (Fig. 4I), regenerative scaffold electrode (RSE) (Fig. 4J) and tissueengineered electronic nerve interface (TEENI) (Fig. 4K) are 


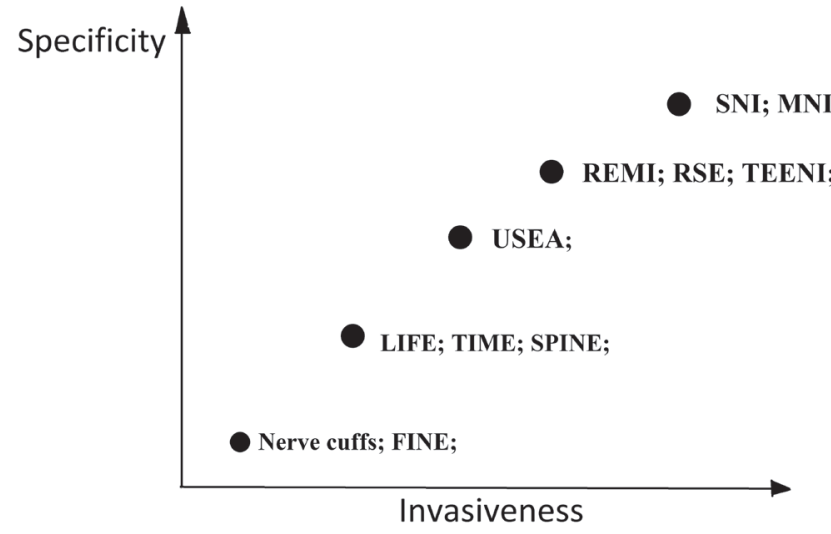

Fig. 5. Comparison of different types of peripheral nerve interfaces based on the level of signal selectivity and interface invasiveness

ideas quite similar to each other. In each of these projects, the 2 ends of the dissected nerve are placed in a special, biocompatible tunnel-shaped dressing. Inside of such a dressing, different types of electrodes can be placed. Regenerating nerve fibers elongate slowly, interacting with the systems in the tunnel and connecting with them. What distinguishes the different approaches is the type of electrodes used. In the REMI project, they look like those from the USEA. The difference is that the nerve grows into the electrodes, as opposed to the electrodes driven into the nerve..$^{49} \mathrm{In}$ RSE, a flat plate with electrodes forms a scaffold inside the tube for nerve development. ${ }^{50}$ Finally, in TEENI, electrodes are loosely suspended in a special hydrogel. This substance contains various elements of the extracellular matrix to support nerve regeneration. Each of these ideas is still being researched and further developed.

Peripheral nerves possess quite a good ability to regenerate, compared to those of central origin. After cutting the nerve, following a period of Wallerian degeneration, it continuously starts to elongate its fibers, looking for a way to re-innervate its target. If the cut nerve stumps are successfully surgically reconnected, its fibers regenerate along endoneural connective tissue channels coated with proliferating Schwann cells (SC) and are guided by neurotrophic factors released by $\mathrm{SC}$ as well as by tropic factors released from the target area. After limb amputation, many muscle groups remain mechanically inactive. For example, the pectoralis major muscle after shoulder disarticulation is no longer able to perform the function of arm adduction. It turns out that it is possible to denervate the muscle (in such case, lateral and medial pectoral nerves can be cleaved) and innervate it again by other nerves (i.e., nerves arising from the lateral, posterior and medial brachial plexus fasciculus, like the musculocutaneous, ulnar, radial, and medial nerve). This procedure is known as targeted muscle re-innervation (TMR) (Fig. 6). As a result, the impulses guided by the motoneurons of the arm are again reflected in motor muscle activity. Briefly, when a patient thinks about arm flexion in his elbow joint, it causes the contraction of muscle fibers of pectoralis major (which are

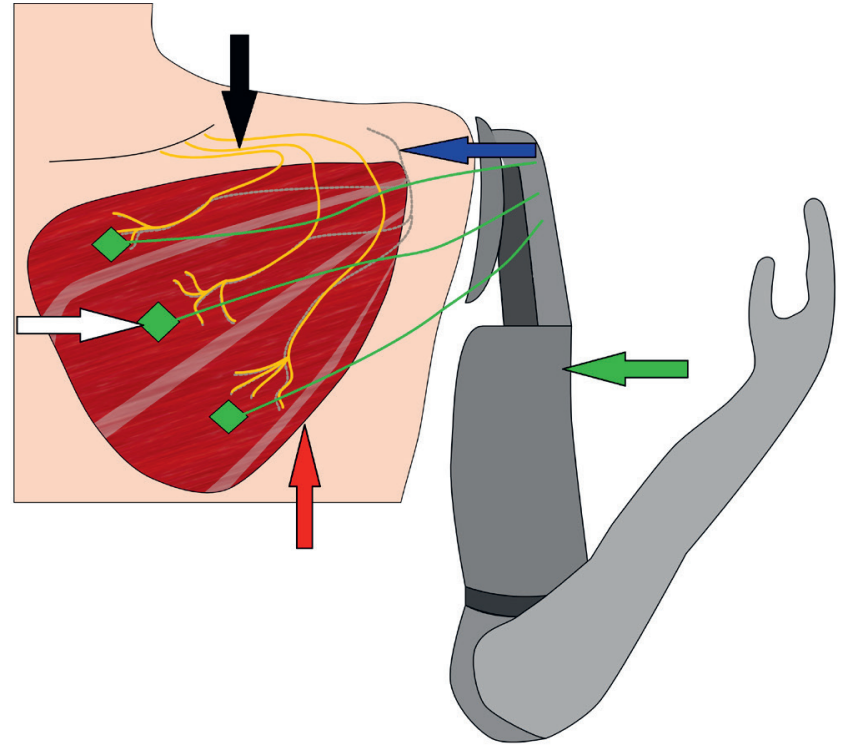

Fig. 6. Scheme of the targeted muscle re-innervation technique. Pectoralis major muscle is denervated, and then re-innervated with a whole set of nerves originating from the brachial plexus - the ulnar median and radial nerves. The signal from the muscle contraction is taken by the electrodes of the electromyograph and converted to signals controlling the artificial limb

Black arrow - brachial plexus; blue arrow - cleaved pectoral nerves, no longer innervating their target; red arrow - pectoralis major muscle; white arrows - electromyographic electrodes; green arrow - artificial limb prosthesis.

innervated by the musculocutaneous nerve). Such signals can be read using electromyographic electrodes and converted into precise signals for moving the prosthesis.

Interestingly, the fields responsible for sensory impressions corresponding to the ulnar, radial and median nerve appear on the skin above the fragments of muscles that underwent neurotization. A patient after TMR, touched at the right place on the skin of the chest, feels it as a touch on the surface of the hand or forearm. The technique of direct muscle neurotization by nerve stumps causes a significant reduction in the occurrence of adverse sensory phenomena after amputation. The feeling of phantom pain and post-amputation pain from neuroma formation often disappears. ${ }^{51}$ This technique, although it has produced quite spectacular effects on prosthesis motor control, provides only a limited number of separate control signals. Therefore, it is still difficult to restore functional degrees of freedom and allow simultaneous independent movements of the fingers, wrists and elbows.

Another approach in this matter, tested so far only in rats, is to place re-growing nerve stumps into synthetic scaffolds along with maturing myoblasts, called regenerative peripheral nerve interface (RPNI). This technique promises more control over the signals from individual nerve fibers, and therefore, more detailed reception of the signals. ${ }^{52}$ It is possible that RPNI will be the key to creating limb prostheses with efficiency comparable to their biological counterparts. 


\section{In vitro biosensors}

Examination of isolated, living nerve cells is tedious work, but necessary for a complete understanding of the physiology of the nervous system. Research of this type is dictated not only by the need to broaden the horizons of neuroscience. Such cells also play a very important role in pharmacological tests, as they make it possible to assess the direct influence of selected chemical substances on the physiological processes occurring in cells. The first step towards research on the activity of individual neurons was the previously-mentioned patch-clamp technique. Limitations connected to it, however, have contributed to the development of other methods of research, each of which is still used in the laboratory environment.

\section{Patch-clamping approach}

The patch-clamp technique was described by Neher and Sakmann in 1976. It uses a micropipette whose end is placed on the surface of the cell membrane. By changing gradients in the concentration of ions across the cell membrane, the micropipette can record the electrical activity of neurons. ${ }^{53}$ The patch-clamping method has made many revolutionary discoveries possible in the field of neuroand electrophysiology. Such a popular research method has tempted scientists to construct a patch-clamp device for the perception of whole neuron systems. As a result, simple neurochips were created using this technique. ${ }^{54}$ This type of technology can be used in laboratories to study neuron physiology and their response to drugs. For this purpose, they are commonly used in laboratories. This concept can also be applied in vivo to analyze patterns of depolarization of single neurons in the brains of laboratory animals.

\section{Multielectrode array}

Multielectrode array (MEA) is a technique usually used for research on neuron physiology. In order to create such a system, nerve cells, most often taken from rats, are cultured on surfaces containing microelectrodes. In this way, researchers can accurately read and stimulate the activity of both individual neurons and entire groups of them. Typically, MEA is a flat chip with a structure reminiscent of a "sandwich". The first, basal layer is basically a dielectric that does not conduct electricity. The next is created with electric conductors that connect to the electrodes in the $3^{\text {rd }}$ layer. The last layer consists again of the dielectric, interrupted by places with flat electrodes coming into contact with the cells. This concept was presented for the first time in $1972 .{ }^{55}$ It is, however, only a general concept that can be interpreted in different ways and supported by many additional research methods (fluorescent calcium indicators, genetic markers and optogenetics). ${ }^{56}$ Multielectrode array is widely used in pharmacology and toxicology. They are successfully used not only for the study of neurons, but also for cardiomyocytes and skeletal muscle. ${ }^{57}$ As part of this technique, it is possible study the activity of both single nerve cells and large tissue structures, such as neural networks.

\section{Neural field effect transistor}

The use of FET (field effect transistor) systems in the integration of neurons with electronic circuits is a derivative of the ISFET (ion-sensitive field effect transistor) technology commonly used in modern medicine. In 1925, the first patent of the key device of the modern era, the FET field transceiver, appeared. The concept was proposed by J.E. Lilienfeld. Later, in 1935, Oskar Heil described the possibility of controlling the resistance in a semiconducting material. Ultimately, the patent for the first fully functional field effect transistor was granted to Nishizawa and Watanabe in 1950. The full understanding of the principles of such a device emerged in 1952 with the work of William Shockley. Briefly, FET consists of 3 electrodes - a source, a drain and a gate, all connected to the channel. Through the source the current enters the transistor, and then it flows through the channel and escapes through the drain. However, this happens only if an appropriate voltage was applied on the gate. If there is no voltage to the gate, the transistor acts as a resistor with very high resistance, which prevents the flow of current. Depending on the method of giving voltage to the gate, the flow of current through the channel can be controlled in various ways. In 1970, Piet Bergveld proposed to use an electrode as the gate that would perceive the electrical voltage resulting from ion concentration in a solution (ISFET). The idea turned out to be revolutionary and is nowadays very widely used in $\mathrm{pH}$ measurements or devices testing the presence of biological molecules (e.g., glucose or DNA). ${ }^{58}$

At the beginning of the 1990s, Fromherz et al. succeeded in connecting an FET system with a living cell. The basic concept behind this project was the idea that a nerve cell could control the gate using the generated electromagnetic field. ${ }^{59}$ Due to the fact that modern computers are based on transistors, it is believed that this technology may prove to be crucial for the effective connection of nervous tissue with an electronic system. Additionally, FET sensor systems that are currently in use can be used in neurophysiological and toxicological studies, and studies of the effects of drugs and the environment on the metabolism of nerve cells. Ultimately, such systems can also be used to study neuronal plasticity and communication between them. ${ }^{60}$

\section{Summary}

In this review paper, we have attempted to briefly present the history and state of the art of the development of methods of connecting the human nervous system with electronic systems for therapeutic purposes. Many of the technological solutions described in this article have 
already been proven as extremely useful in contemporary neurorestorative medicine. Brain-computer interfaces, both non-invasive and invasive, play an important role in the rehabilitation of people with severe movement impairment. Patients with severe visual and hearing impairment benefit from retinal and cochlear implants and can lead a relatively normal life. The spectacular success of the targeted muscle re-innervation technique has led to improvement in the life of quality of people after upper limb amputation.

Our analysis of the variety of available technical solutions has enabled us to determine the properties of the ideal interface between the nervous system and the electronic system. This interface should be characterized by high sensitivity and resolution of the received neuronal signals, and minimal invasiveness of the technique in its in vivo implantation as well as high and long-term biocompatibility. We believe that it will be possible to develop interfaces that would fully decode central or peripheral neuronal activity for the treatment of spinal cord injuries and other lesions of the CNS and for ideal control of artificial limb prostheses. In addition, the work of scientists may soon provide a stable interface that allows a person to communicate with a computer with just a simple thought.

\section{ORCID iDs}

Tymoteusz Skok (1) https://orcid.org/0000-0001-9094-9620 Paweł Tabakow (1) https://orcid.org/0000-0001-8638-3871 Krzysztof Chmielak (1) https://orcid.org/0000-0001-9383-7667

\section{References}

1. Cobb M. Timeline: Exorcizing the animal spirits. Jan Swammerdam on nerve function. Nat Rev Neurosci. 2002;3(5):395-400.

2. Sleigh C. Jan Swammerdam's frogs. Notes Rec R Soc Lond. 2012;66(4): 373-392.

3. Verkhratsky A, Parpura V. History of electrophysiology and the patch clamp. Methods Mol Biol. 2014;1183:1-19.

4. Wallace W. The vibrating nerve impulse in Newton, Willis and Gassendi: First steps in a mechanical theory of communication. Brain Cogn. 2003;51(1):66-94.

5. Verkhratsky A, Krishtal OA, Petersen OH. From Galvani to patch clamp: The development of electrophysiology. Pflugers Arch. 2006;453(3): 233-247.

6. Harrar JE. The potentiostat and the voltage clamp. Electrochem Soc Interface. 2013;22(4):42-44.

7. McNamee MJ, Edwards SD. Transhumanism, medical technology and slippery slopes. J Med Ethics. 2006;32(9):513-518.

8. Mudry A, Mills M. The early history of the cochlear implant: A retrospective. JAMA Otolaryngol Head Neck Surg. 2013;139(5):446-453.

9. Durand DM. What is neural engineering? J Neural Eng. 2006;4(4). doi:10.1088/1741-2552/4/4/e01

10. Finkel LH. Neuroengineering models of brain disease. Annu Rev Biomed Eng. 2000;2(1):577-606.

11. Vidal JJ. Real-time detection of brain events in EEG. Proc IEEE Inst Electr Electron Eng. 1977;65(5):633-641.

12. Shih JJ, Krusienski DJ, Wolpaw JR. Brain-computer interfaces in medicine. Mayo Clin Proc. 2012;87(3):268-279.

13. Anupama HS, Cauvery NK, Lingaraju GM. Brain computer interface and its types - a study. IJASEAT. 2012;3(2):739-745.

14. Nezamfar H, Salehi SSM, Moghadamfalahi M, Erdogmus D. A context-aware c-VEP-based BCI typing interface using EEG signals. IEEE J-STSP. 2016;10(5):932-941.
15. Kaufmann T, Herweg A, Kübler A. Toward brain-computer interface based wheelchair control utilizing tactually-evoked event-related potentials. J Neuroeng Rehabil. 2014;11:7.

16. Sirvent JL, Azorín JM, láñez E, Úbeda A, Fernández E. P300-based brain-computer interface for internet browsing. In: Trends in Practical Applications of Agents and Multiagent Systems. Advances in Intelligent and Soft Computing. $8^{\text {th }}$ International Conference on Practical Applications of Agents and Multiagent Systems. 2010;615-622.

17. Pires G, Torres M, Casaleiro N, Nunes U, Castelo-Branco M. Playing Tetris with non-invasive $\mathrm{BCI}$. Paper presented at: the $2011 \mathrm{IEEE} 1^{\text {st }}$ International Conference on Serious Games and Applications for Health (SeGAH); November 16-18 2011; Braga, Portugal.

18. Spüler M. A high-speed brain-computer interface (BCl) using dry EEG electrodes. PLoS One. 2017;12(2):e0172400.

19. Sitaram R, Caria A, Veit R, et al. fMRI brain-computer interface: $A$ tool for neuroscientific research and treatment. Comput Intell Neurosci. 2007;2007:25487.

20. Mellinger J, Schalk G, Braun C, et al. An MEG-based brain-computer interface (BCI). Neuroimage. 2007;36(3):581-593.

21. Schalk G, Leuthardt EC. Brain-computer interfaces using electrocorticographic signals. IEEE Rev Biomed Eng. 2011;4:140-154.

22. Liu J, Fu T-M, Cheng Z, et al. Syringe-injectable electronics. Nat Nanotechnology. 2015;10(7):629-636.

23. Kennedy PR, Bakay RA. Restoration of neural output from a paralyzed patient by a direct brain connection. Neuroreport. 1998;9(8): 1707-1711.

24. Brower V. When mind meets machine. EMBO Rep. 2005;6(2):108-110.

25. Collinger JL, Wodlinger B, Downey JE, et al. High-performance neuroprosthetic control by an individual with tetraplegia. Lancet. 2013; 381(9866):557-564.

26. Jarosiewicz B, Sarma AA, Bacher D, et al. Virtual typing by people with tetraplegia using a self-calibrating intracortical brain-computer interface. Sci Transl Med. 2015;7(313):313ra179.

27. Ludwig KA. Neuroprosthetic Devices: Inputs and Outputs. Ph.D. Thesis, University of Michigan, Ann Arbor, MI, USA, 2009.

28. Capogrosso M, Milekovic T, Borton, D, et al. A brain-spine interface alleviating gait deficits after spinal cord injury in primates. Nature. 2016;539(7628):284-288.

29. Bouton CE, Shaikhouni A, Annetta NV, et al. Restoring cortical control of functional movement in a human with quadriplegia. Nature. 2016;533(7602):247-250.

30. Dobelle WH. Artificial vision for the blind by connecting a television camera to the visual cortex. ASAIO J. 2000;46(1):3-9.

31. Rachitskaya AV, Yuan A, Marino MJ, Reese J, Ehlers JP. Intraoperative OCT imaging of the Argus II Retinal Prosthesis System. Ophthalmic Surg Lasers Imaging Retina. 2016;47(11):999-1003.

32. Stingl K, Bartz-Schmidt KU, Besch D, et al. Subretinal Visual Implant Alpha IMS: Clinical trial interim report. Vision Res. 2015;111(Pt B): 149-160.

33. Berger TW, Song D, Chan RHM, et al. A hippocampal cognitive prosthesis: Multi-input, multi-output nonlinear modeling and VLSI implementation. IEEE Trans Neural Syst Rehabil Eng. 2012;20(2):198-211.

34. Griffith RW, Humphrey DR. Long-term gliosis around chronically implanted platinum electrodes in the Rhesus macaque motor cortex. Neurosci Lett. 2006;406(1-2):81-86.

35. Waldert S. Invasive vs non-invasive neuronal signals for brain-machine interfaces: Will one prevail? Front Neurosci. 2016;10:295.

36. Tyler DJ, Polasek KH, Schiefer MA. Chapter 63 - Peripheral nerve interfaces. In: Tubbs R, Rizk E, Shoja MM, Loukas M, Barbaro N, Spinner RJ, eds. Nerves and Nerve Injuries. San Diego, CA: Academic Press; 2015: 1033-1054.

37. Spearman BS, Desai VH, Mobini S, et al. Neural interfaces: Tissueengineered peripheral nerve interfaces. Adv Funct Mater. 2018;28(12): 1870076. doi:10.1002/adfm.201870076

38. Naples GG, Mortimer JT, Scheiner A, Sweeney JD. A spiral nerve cuff electrode for peripheral nerve stimulation. IEEE Trans Biomed Eng. 1988;35(11):905-916.

39. Foldes EL, Ackermann DM, Bhadra N, Kilgore KL, Bhadra N. Design, fabrication and evaluation of a conforming circumpolar peripheral nerve cuff electrode for acute experimental use. J Neurosci Methods. 2011;196(1):31-37. 
40. Tyler DJ, Durand DM. Chronic response of the rat sciatic nerve to the flat interface nerve electrode. Ann Biomed Eng. 2003;31(6):633-642.

41. Dweiri YM, Stone MA, Tyler DJ, McCallum GA, Durand DM. Fabrication of high contact-density, flat-interface nerve electrodes for recording and stimulation applications. J Vis Exp. 2016;4(116).

42. Yoshida K, Hennings K, Kammer S. Acute performance of the thin-film longitudinal intra-fascicular electrode. Paper presented at: the First IEEE/RAS-EMBS International Conference on Biomedical Robotics and Biomechatronics (BioRob 2006); February 20-22, 2006; Pisa, Italy.

43. Boretius T, Badia J, Pascual-Font A, et al. A transverse intrafascicular multichannel electrode (TIME) to interface with the peripheral nerve. Biosens Bioelectron. 2010;26(1):62-69.

44. Yoshida K, Stieglitz T, Qiao S. Bioelectric interfaces for the periph eral nervous system. Paper presented at: the $36^{\text {th }}$ Annual International Conference of the IEEE Engineering in Medicine and Biology Society; August 26-30 2014; Chicago, IL.

45. Tyler DJ, Durand DM. A slowly penetrating interfascicular nerve electrode for selective activation of peripheral nerves. IEEE Trans Rehabil Eng. 1997;5(1):51-61.

46. Clark GA, Ledbetter NM, Warren DJ, Harrison RR. Recording sensory and motor information from peripheral nerves with Utah slanted electrode arrays. ConfProc IEEE Eng Med Biol Soc. 2011;2011:4641-4644.

47. Birenbaum NK, MacEwan MR, Ray WZ. Interfacing peripheral nerve with macro-sieve electrodes following spinal cord injury. Neural Regen Res. 2017;12(6):906-909.

48. Musick KM, Rigosa J, Narasimhan S, et al. Chronic multichannel neural recordings from soft regenerative microchannel electrodes during gait. Sci Rep. 2015;5:14363.

49. Lotfi P, Garde K, Chouhan A, Bengali E, Romero M. Modality-specific axonal regeneration: Toward selective regenerative neural interfaces. Front Neuroeng. 2011;4:11.
50. Clements IP, Mukhatyar VJ, Srinivasan A, Bentley JT, Andreasen DS, Bellamkonda RV. Regenerative scaffold electrodes for peripheral nerve interfacing. IEEE Trans Neural Syst Rehabil Eng. 2013;21(4):554-566.

51. Cheesborough JE, Smith LH, Kuiken TA, Dumanian GA. Targeted muscle reinnervation and advanced prosthetic arms. Semin Plast Surg. 2015;29(1):62-72.

52. Urbanchek MG, Kung TA, Frost CM, et al. Development of a regenerative peripheral nerve interface for control of a neuroprosthetic limb. Biomed Res Int. 2016;12:1-8.

53. Kornreich BG. The patch clamp technique: Principles and technical considerations. J Vet Cardiol. 2007;9(1):25-37

54. Py C, Denhoff MW, Sabourin N, Weber J, Shiu M, Zhao P. Priming and testing silicon patch-clamp neurochips. N Biotechnol. 2014;31(5): 430-435.

55. Pine J. A history of MEA development. In: Taketani M, Baudry M, eds. Advances in Network Electrophysiology: Using Multi-Electrode Arrays. Boston, MA: Springer US; 2006:3-23.

56. Rutten W, Mouveroux JM, Buitenweg J, et al. Neuroelectronic interfacing with cultured multielectrode arrays toward a cultured probe. Proc IEEE Inst Electr Electron Eng. 2001;89(7):1013-1029.

57. Rabieh N, Ojovan SM, Shmoel N, Erez, H, Maydan E, Spira ME. Onchip, multisite extracellular and intracellular recordings from primary cultured skeletal myotubes. Scientific Reports. 2016;6:36498.

58. Walsh KB, DeRoller N, Zhu Y, Koley G. Application of ion-sensitive field effect transistors for ion channel screening. Biosens Bioelectron. 2014;54:448-454.

59. Fromherz P, Offenhausser A, Vetter T, Weis J. A neuron-silicon junction: A Retzius cell of the leech on an insulated-gate field-effect transistor. Science. 1991;252(5010):1290-1293.

60. Liu Q, Wu C, Cai H, Hu N, Zhou J, Wang P. Cell-based biosensors and their application in biomedicine. Chem Rev. 2014;114(12):6423-6461. 